\title{
Neutrino Masses from Broken R-Parity
}

\author{
J. C. Romão $o^{\mathrm{a}}$ \\ ${ }^{a}$ Instituto Superior Técnico, Departamento de Física \\ A. Rovisco Pais 1, 1049-001 Lisboa, Portugal
}

We review models where $\mathrm{R}$-parity is broken, either spontaneously or explicitly. In this last case we consider the situation where $\mathrm{R}$-parity is broken via bilinear terms in the superpotential. We show that although at tree level only one neutrino gets mass, at one-loop level all three neutrinos became massive. We study the conditions under which bimaximal mixing can be achieved and show that the masses can be in the correct ranges needed for solving the atmospheric and solar neutrino problems.

\section{Introduction}

In the past most discussions of supersymmetric (SUSY) [1,2] phenomenology assumed R-parity $\left(R_{P}\right)$ conservation where,

$R_{P}=(-1)^{2 J+3 B+L}$

This implies that SUSY particles are pair produced, every SUSY particle decays into another SUSY particle and that there is a $L S P$ that it is stable. But this is just an ad hoc assumption without a deep justification. In this talk we will review how $R_{P}$ can be broken, either spontaneously or explicitly, and discuss the most important features of these models [3]. We will also describe recent results [4] on one-loop generated masses and mixings in the context of a model that is a minimal extension of the minimal extension of the MSSM-GUT [5] in which $R_{P}$ Violation (RPV) is introduced via a bilinear term in the MSSM superpotential [6,7].

\section{Spontaneously Broken R-Parity}

\subsection{The Original Proposal}

In the original proposal [8] the content was just the MSSM and the breaking was induced by

$\left\langle\tilde{\nu}_{\tau}\right\rangle=v_{L}$

The problem with this model was that the Majoron $J$ coupled to $Z^{0}$ with gauge strength and

*This work was supported by the TMR network grant ERBFMRXCT960090 of the European Union.
Table 1

Lepton number assignments.

\begin{tabular}{lccccc}
\hline Field & $L$ & $e^{c}$ & $\nu^{c}$ & $S$ & others \\
Lepton \# & 1 & -1 & -1 & 1 & 0 \\
\hline
\end{tabular}

therefore the decay $Z^{0} \rightarrow \rho_{L} J$ contributed to the invisible $Z$ width the equivalent of half a (light) neutrino family. After LEP I this was excluded.

\subsection{A Viable Model for SBRP}

The way to avoid the previous difficulty is to enlarge the model and make $J$ mostly out of isosinglets. This was proposed by Masiero and Valle [9]. The content is the MSSM plus a few Isosinglet Superfields that carry lepton number,

$\nu_{i}^{c} \equiv(1,0,-1) ; S_{i} \equiv(1,0,1) ; \Phi \equiv(1,0,0)$

The model is defined by the superpotential [9, 10],

$$
\begin{aligned}
W= & h_{u} u^{c} Q H_{u}+h_{d} d^{c} Q H_{d}+h_{e} e^{c} L H_{d} \\
& +\left(h_{0} H_{u} H_{d}-\mu^{2}\right) \Phi \\
& +h_{\nu} \nu^{c} L H_{u}+h \Phi \nu^{c} S
\end{aligned}
$$

where the lepton number assignments are shown in Table 1. The spontaneous breaking of $\mathrm{R}$ parity and lepton number is driven by 10 ]

$v_{R}=\left\langle\tilde{\nu}_{R \tau}\right\rangle \quad v_{S}=\left\langle\tilde{S}_{\tau}\right\rangle \quad v_{L}=\left\langle\tilde{\nu}_{\tau}\right\rangle$

The electroweak breaking and fermion masses arise from

$$
\left\langle H_{u}\right\rangle=v_{u} \quad\left\langle H_{d}\right\rangle=v_{d}
$$


with $v^{2}=v_{u}^{2}+v_{d}^{2}$ fixed by the $\mathrm{W}$ mass. The Majoron is given by the imaginary part of

$\frac{v_{L}^{2}}{V v^{2}}\left(v_{u} H_{u}-v_{d} H_{d}\right)+\frac{v_{L}}{V} \tilde{\nu_{\tau}}-\frac{v_{R}}{V} \nu^{\tilde{c}_{\tau}}+\frac{v_{S}}{V} \tilde{S}_{\tau}(6)$

where $V=\sqrt{v_{R}^{2}+v_{S}^{2}}$. Since the Majoron is mainly an $S U(2) \otimes U(1)$ singlet it does not contribute to the invisible $Z^{0}$ decay width.

\subsection{Some Results on SBRP}

The SBRP model has been extensively studied. The implications for accelerator [11] and non-accelerator [12] physics have been presented before and we will not discuss them here [3]. As in this talk we are concerned with the neutrino properties in the context of $R_{P}$ models we will only review here the neutrino results.

\section{- Neutrinos have mass}

Neutrinos are massless at Lagrangian level but get mass from the mixing with neutralinos [13,14]. In the SBRP model it is possible to have non zero masses for two neutrinos [14.

- Neutrinos mix

The mixing is related to the the coupling matrix $h_{\nu_{i j}}$. This matrix has to be non diagonal in generation space to allow

$\nu_{\tau} \rightarrow \nu_{\mu}+J$

and therefore evading [14] the Critical Density Argument against $\nu^{\prime} s$ in the $\mathrm{MeV}$ range.

- Avoiding BBN constraints on the $m_{\nu_{\tau}}$

In the $S M \mathrm{BBN}$ arguments [15] rule out $\nu_{\tau}$ masses in the range

$0.5 \mathrm{MeV}<m_{\nu_{\tau}}<35 \mathrm{MeV}$

We have shown [16] that $S B R P$ models can evade that constraint due to new annihilation channels

$\nu_{\tau} \nu_{\tau} \rightarrow J J$

\section{Explicitly Broken R-Parity}

The most general superpotential $W$ with the particle content of the MSSM is given by [6.7]

$W=W_{M S S M}+W_{R}$

where

$$
\begin{aligned}
W_{M S S M}=\varepsilon_{a b} & {\left[h_{U}^{i j} \widehat{Q}_{i}^{a} \widehat{U}_{j} \widehat{H}_{u}^{b}+h_{D}^{i j} \widehat{Q}_{i}^{b} \widehat{D}_{j} \widehat{H}_{d}^{a}\right.} \\
+ & \left.h_{E}^{i j} \widehat{L}_{i}^{b} \widehat{R}_{j} \widehat{H}_{d}^{a}-\mu \widehat{H}_{d}^{a} \widehat{H}_{u}^{b}\right]
\end{aligned}
$$

and

$$
\begin{aligned}
W_{R}= & \varepsilon_{a b}\left[\lambda_{i j k} \widehat{L}_{i}^{a} \widehat{L}_{j}^{b} \widehat{R}_{k}+\lambda_{i j k}^{\prime} \widehat{D}_{i} \widehat{L}_{j}^{a} \widehat{Q}_{k}^{b}\right] \\
& +\lambda_{i j k}^{\prime \prime} \widehat{D}_{i} \widehat{D}_{j} \widehat{U}_{k}+\varepsilon_{a b} \epsilon_{i} \widehat{L}_{i}^{a} \widehat{H}_{u}^{b}
\end{aligned}
$$

where $i, j=1,2,3$ are generation indices, $a, b=$ 1,2 are $S U(2)$ indices.

The set of soft supersymmetry breaking terms are

$$
\begin{aligned}
V^{\text {soft }}= & V_{M S S M}^{\text {soft }}+V_{\mathbb{R}}^{\text {soft }} \\
V_{\mathrm{MSSM}}^{\text {soft }}= & M_{Q}^{i j 2} \widetilde{Q}_{i}^{a *} \widetilde{Q}_{j}^{a}+M_{U}^{i j 2} \widetilde{U}_{i}^{*} \widetilde{U}_{j}+M_{D}^{i j 2} \widetilde{D}_{i}^{*} \widetilde{D}_{j} \\
& +M_{L}^{i j 2} \widetilde{L}_{i}^{a *} \widetilde{L}_{j}^{a}+M_{R}^{i j 2} \widetilde{R}_{i}^{*} \widetilde{R}_{j} \\
& +m_{H_{d}}^{2} H_{d}^{a *} H_{d}^{a}+m_{H_{u}}^{2} H_{u}^{a *} H_{u}^{a} \\
& -\left[\frac{1}{2} \sum_{i=1}^{3} M_{i} \lambda_{i} \lambda_{i}+h . c .\right] \\
& +\varepsilon_{a b}\left[A_{U}^{i j} \widetilde{Q}_{i}^{a} \widetilde{U}_{j} H_{u}^{b}+A_{D}^{i j} \widetilde{Q}_{i}^{b} \widetilde{D}_{j} H_{d}^{a}\right. \\
& \left.+A_{E}^{i j} \widetilde{L}_{i}^{b} \widetilde{R}_{j} H_{d}^{a}-B \mu H_{d}^{a} H_{u}^{b}+h . c .\right]
\end{aligned}
$$

and

$$
\begin{aligned}
V_{R}^{\text {soft }}= & \varepsilon_{a b}\left[A_{\lambda}^{i j} \widetilde{L}_{i}^{a} \widetilde{L}_{j}^{b} \widetilde{R}_{k}+A_{\lambda^{\prime}}^{i j k} \widetilde{D}_{i} \widetilde{L}_{j}^{a} \widetilde{Q}_{k}^{b}\right] \\
& +A_{\lambda^{\prime \prime}}^{i j} \widetilde{D}_{i} \widetilde{D}_{j} \widetilde{U}_{k}+\varepsilon_{a b} B_{i} \epsilon_{i} \widetilde{L}_{i}^{a} H_{u}^{b}+h . c
\end{aligned}
$$

The bilinear $R_{P}$ violating term cannot be eliminated by superfield redefinition as sometimes it is claimed. To show this we consider the case 17] where all the trilinear couplings in Eq. (12) are zero and for simplicity we take $\epsilon_{1}=\epsilon_{2}=0$. Then the superpotential is

$$
W=\varepsilon_{a b}\left[h_{t} \widehat{Q}_{3}^{a} \widehat{U}_{3} \widehat{H}_{u}^{b}+h_{b} \widehat{Q}_{3}^{b} \widehat{D}_{3} \widehat{H}_{d}^{a}+h_{\tau} \widehat{L}_{3}^{b} \widehat{R}_{3} \widehat{H}_{d}^{a}\right.
$$




$$
\left.-\mu \widehat{H}_{d}^{a} \widehat{H}_{u}^{b}+\epsilon_{3} \widehat{L}_{3}^{a} \widehat{H}_{u}^{b}\right]
$$

Consider now the rotation

$\widehat{H}_{d}^{\prime}=\frac{\mu \widehat{H}_{d}-\epsilon_{3} \widehat{L}_{3}}{\sqrt{\mu^{2}+\epsilon_{3}^{2}}}, \quad \widehat{L}_{3}^{\prime}=\frac{\epsilon_{3} \widehat{H}_{d}+\mu \widehat{L}_{3}}{\sqrt{\mu^{2}+\epsilon_{3}^{2}}}$

In the new basis

$$
\begin{aligned}
W= & h_{t} \widehat{Q}_{3} \widehat{U}_{3} \widehat{H}_{u}+h_{b} \frac{\mu}{\mu^{\prime}} \widehat{Q}_{3} \widehat{D}_{3} \widehat{H}_{d}^{\prime}+h_{\tau} \widehat{L}_{3}^{\prime} \widehat{R}_{3} \widehat{H}_{d}^{\prime} \\
& -\mu^{\prime} \widehat{H}_{d}^{\prime} \widehat{H}_{u}+h_{b} \frac{\epsilon_{3}}{\mu^{\prime}} \widehat{Q}_{3} \widehat{D}_{3} \widehat{L}_{3}^{\prime}
\end{aligned}
$$

where

$\mu^{\prime 2}=\mu^{2}+\epsilon_{3}^{2}$

But the soft terms,

$$
\begin{aligned}
V_{\text {soft }}= & m_{H_{d}}^{2}\left|H_{d}\right|^{2}+M_{L_{3}}^{2}\left|\widetilde{L}_{3}\right|^{2} \\
& +\left[B \mu H_{d} H_{u}-B_{2} \epsilon_{3} \widetilde{L}_{3} H_{u}+\text { h.c. }\right] \\
& +\cdots
\end{aligned}
$$

in the rotated basis is

$$
\begin{aligned}
V_{\text {sof } t} & =\frac{m_{H_{d}}^{2} \mu^{2}+M_{L_{3}}^{2} \epsilon_{3}^{2}}{\mu^{\prime 2}}\left|H_{d}^{\prime}\right|^{2}+\frac{m_{H_{d}}^{2} \epsilon_{3}^{2}+M_{L_{3}}^{2} \mu^{2}}{\mu^{\prime 2}}\left|\widetilde{L}_{3}^{\prime}\right|^{2} \\
& -\left[\frac{B \mu^{2}+B_{2} \epsilon_{3}^{2}}{\mu^{\prime}} H_{d}^{\prime} H_{u}-\frac{\epsilon_{3} \mu}{\mu^{\prime 2}}\left(m_{H_{d}}^{2}-M_{L_{3}}^{2} \widetilde{L}_{3}^{\prime} H_{d}^{\prime}\right.\right. \\
& \left.-\frac{\epsilon_{3} \mu}{\mu^{\prime}}\left(B_{2}-B\right) \widetilde{L}_{3}^{\prime} H_{u}+\text { h.c. }\right]+\cdots
\end{aligned}
$$

The last two terms violate $R_{P}$ and induce a nonzero VEV for the $\tau$ sneutrino field in the rotated basis $\left\langle\tilde{\nu}_{\tau}^{\prime}\right\rangle=v_{3}^{\prime} / \sqrt{2}$, where

$v_{3}^{\prime} \approx-\frac{\epsilon_{3} \mu}{\mu^{\prime 2} m_{\tilde{\nu}_{\tau}^{0}}^{2}}\left(v_{1}^{\prime} \Delta m^{2}+\mu^{\prime} v_{2} \Delta B\right)$

and

$$
\begin{aligned}
\Delta m^{2} & \equiv m_{H_{1}}^{2}-M_{L_{3}}^{2} \\
& \approx-\frac{3 h_{b}^{2}}{8 \pi^{2}}\left(m_{H_{1}}^{2}+M_{Q}^{2}+M_{D}^{2}+A_{D}^{2}\right) \ln \frac{M_{G U T}}{m_{Z}} \\
\Delta B & \equiv B_{2}-B \approx \frac{3 h_{b}^{2}}{8 \pi^{2}} A_{D} \ln \frac{M_{G U T}}{m_{Z}}
\end{aligned}
$$

\section{Bilinear R-Parity Violation}

\subsection{The Model}

The superpotential $W$ for the bilinear $R_{P}$ violation model is given by [6, [7]

$$
\begin{gathered}
W=\varepsilon_{a b}\left[h_{U}^{i j} \widehat{Q}_{i}^{a} \widehat{U}_{j} \widehat{H}_{u}^{b}+h_{D}^{i j} \widehat{Q}_{i}^{b} \widehat{D}_{j} \widehat{H}_{d}^{a}+h_{E}^{i j} \widehat{L}_{i}^{b} \widehat{R}_{j} \widehat{H}_{d}^{a}\right. \\
\left.-\mu \widehat{H}_{d}^{a} \widehat{H}_{u}^{b}+\epsilon_{i} \widehat{L}_{i}^{a} \widehat{H}_{u}^{b}\right]
\end{gathered}
$$

where $i, j=1,2,3$ are generation indices, $a, b=$ 1,2 are $S U(2)$ indices. The set of soft supersymmetry breaking terms are

$$
\begin{aligned}
V_{\text {soft }}= & M_{Q}^{i j 2} \widetilde{Q}_{i}^{a *} \widetilde{Q}_{j}^{a}+M_{U}^{i j 2} \widetilde{U}_{i}^{*} \widetilde{U}_{j}+M_{D}^{i j 2} \widetilde{D}_{i}^{*} \widetilde{D}_{j} \\
& +M_{L}^{i j 2} \widetilde{L}_{i}^{a *} \widetilde{L}_{j}^{a}+M_{R}^{i j 2} \widetilde{R}_{i}^{*} \widetilde{R}_{j}+m_{H_{d}}^{2} H_{d}^{a *} H_{d}^{a} \\
& +m_{H_{u}}^{2} H_{u}^{a *} H_{u}^{a}-\left[\frac{1}{2} \sum M_{i} \lambda_{i} \lambda_{i}+h . c .\right] \\
& +\varepsilon_{a b}\left[A_{U}^{i j} \widetilde{Q}_{i}^{a} \widetilde{U}_{j} H_{u}^{b}+A_{D}^{i j} \widetilde{Q}_{i}^{b} \widetilde{D}_{j} H_{d}^{a}\right. \\
& \left.+A_{E}^{i j} \widetilde{L}_{i}^{b} \widetilde{R}_{j} H_{d}^{a}-B \mu H_{d}^{a} H_{u}^{b}+B_{i} \epsilon_{i} \widetilde{L}_{i}^{a} H_{u}^{b}\right] .
\end{aligned}
$$

The electroweak symmetry is broken when the VEVS of the two Higgs doublets $H_{d}$ and $H_{u}$, and the sneutrinos.

$$
\begin{aligned}
H_{d} & =\left(\begin{array}{c}
\frac{1}{\sqrt{2}}\left[\chi_{d}^{0}+v_{d}+i \varphi_{d}^{0}\right] \\
H_{d}^{-}
\end{array}\right) \\
H_{u} & =\left(\begin{array}{c}
H_{u}^{+} \\
\frac{1}{\sqrt{2}}\left[\chi_{u}^{0}+v_{u}+i \varphi_{u}^{0}\right]
\end{array}\right) \\
L_{i} & =\left(\begin{array}{c}
\frac{1}{\sqrt{2}}\left[\tilde{\nu}_{i}^{R}+v_{i}+i \tilde{\nu}_{i}^{I}\right] \\
\tilde{\ell}^{i}
\end{array}\right)
\end{aligned}
$$

The gauge bosons $W$ and $Z$ acquire masses

$$
m_{W}^{2}=\frac{1}{4} g^{2} v^{2} \quad ; \quad m_{Z}^{2}=\frac{1}{4}\left(g^{2}+g^{\prime 2}\right) v^{2}
$$

where

$$
v^{2} \equiv v_{d}^{2}+v_{u}^{2}+v_{1}^{2}+v_{2}^{2}+v_{3}^{2}=(246 \mathrm{GeV})^{2}
$$

We introduce the following notation in spherical coordinates:

$$
\begin{aligned}
v_{d} & =v \sin \theta_{1} \sin \theta_{2} \sin \theta_{3} \cos \beta \\
v_{u} & =v \sin \theta_{1} \sin \theta_{2} \sin \theta_{3} \sin \beta \\
v_{1} & =v \sin \theta_{1} \sin \theta_{2} \cos \theta_{3}
\end{aligned}
$$


$v_{2}=v \sin \theta_{1} \cos \theta_{2}$

$v_{3}=v \cos \theta_{1}$

which preserves the MSSM definition $\tan \beta=$ $v_{u} / v_{d}$. The angles $\theta_{i}$ are equal to $\pi / 2$ in the MSSM limit.

The full scalar potential may be written as

$V_{\text {total }}=\sum_{i}\left|\frac{\partial W}{\partial z_{i}}\right|^{2}+V_{D}+V_{\text {soft }}+V_{R C}$

where $z_{i}$ denotes any one of the scalar fields in the theory, $V_{D}$ are the usual $D$-terms, $V_{\text {soft }}$ the SUSY soft breaking terms, and $V_{R C}$ are the oneloop radiative corrections.

In writing $V_{R C}$ we use the diagrammatic method and find the minimization conditions by correcting to one-loop the tadpole equations. This method has advantages with respect to the effective potential when we calculate the one-loop corrected scalar masses. The scalar potential contains linear terms

$V_{\text {linear }}=t_{d} \sigma_{d}^{0}+t_{u} \sigma_{u}^{0}+t_{i} \tilde{\nu}_{i}^{R} \equiv t_{\alpha} \sigma_{\alpha}^{0}$,

where we have introduced the notation

$\sigma_{\alpha}^{0}=\left(\sigma_{d}^{0}, \sigma_{u}^{0}, \nu_{1}^{R}, \nu_{2}^{R}, \nu_{3}^{R}\right)$

and $\alpha=d, u, 1,2,3$. The one loop tadpoles are

$$
\begin{aligned}
t_{\alpha} & =t_{\alpha}^{0}-\delta t_{\alpha}^{\overline{M S}}+T_{\alpha}(Q) \\
& =t_{\alpha}^{0}+T_{\alpha}^{\overline{M S}}(Q)
\end{aligned}
$$

where $T_{\alpha}^{\overline{M S}}(Q) \equiv-\delta t_{\alpha}^{\overline{M S}}+T_{\alpha}(Q)$ are the finite one-loop tadpoles.

\subsection{Main Features}

The $\epsilon$-model is a one (three) parameter(s) generalization of the MSSM. It can be thought as an effective model showing the more important features of the SBRP-model [10] at the weak scale. The mass matrices, charged and neutral currents, are similar to the SBRP-model if we identify

$\epsilon \equiv v_{R} h_{\nu}$

The $R_{P}$ violating parameters $\epsilon_{i}$ and $v_{i}$ violate lepton number, inducing a non-zero mass for only one neutrino, which could be considered to be the

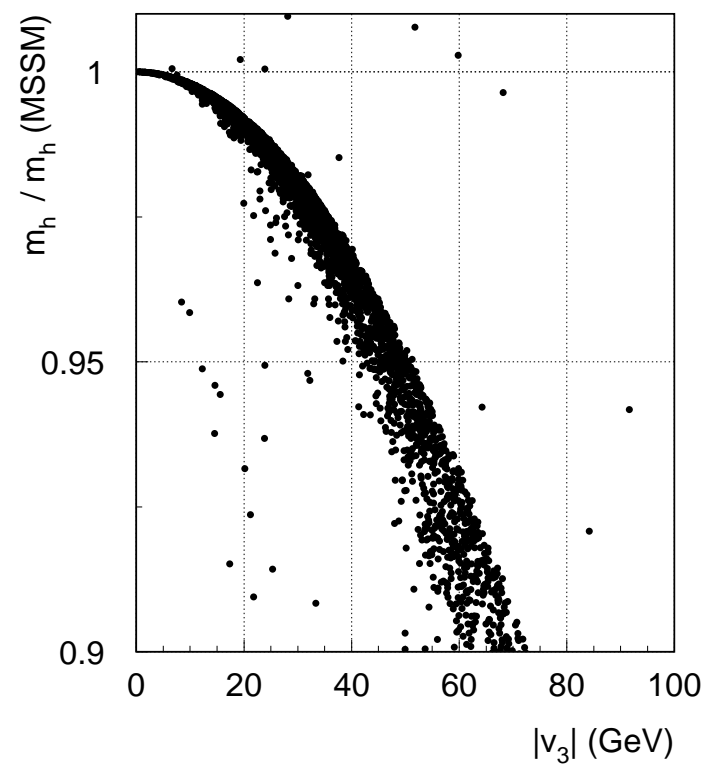

Figure 1. Ratio of the lightest CP-even Higgs boson mass $m_{h}$ in the $\epsilon$-model and in the MSSM as a function of $v_{3}$.

the $\nu_{\tau}$. The $\nu_{e}$ and $\nu_{\mu}$ remain massless in first approximation. As we will explain below, they acquire masses from supersymmetric loops [4, 18] that are typically smaller than the tree level mass.

The model has the MSSM as a limit. This can be illustrated in Figure 1 where we show the ratio of the lightest CP-even Higgs boson mass $m_{h}$ in the $\epsilon$-model and in the MSSM as a function of $v_{3}$. Many other results concerning this model and the implications for physics at the accelerators can be found in ref. [6,:].

\section{Radiative Breaking}

\subsection{Radiative Breaking in the $\epsilon$ model: The minimal case}

At $Q=M_{G U T}$ we assume the standard minimal supergravity unifications assumptions,

$$
\begin{aligned}
& A_{t}=A_{b}=A_{\tau} \equiv A, \\
& B=B_{2}=A-1, \\
& m_{H_{d}}^{2}=m_{H_{u}}^{2}=M_{L}^{2}=M_{R}^{2}=m_{0}^{2},
\end{aligned}
$$




$$
\begin{aligned}
& M_{Q}^{2}=M_{U}^{2}=M_{D}^{2}=m_{0}^{2}, \\
& M_{3}=M_{2}=M_{1}=M_{1 / 2}
\end{aligned}
$$

In order to determine the values of the Yukawa couplings and of the soft breaking scalar masses at low energies we first run the RGE's from the unification scale $M_{G U T} \sim 10^{16} \mathrm{GeV}$ down to the weak scale. We randomly give values at the unification scale for the parameters of the theory.

$$
\begin{gathered}
10^{-2} \leq h_{t G U T}^{2} / 4 \pi \leq 1 \\
10^{-5} \leq h_{b G U T}^{2} / 4 \pi \leq 1 \\
-3 \leq A / m_{0} \leq 3 \\
0 \leq \mu_{G U T}^{2} / m_{0}^{2} \leq 10 \\
0 \leq M_{1 / 2} / m_{0} \leq 5 \\
10^{-2} \leq \epsilon_{\text {GUT }}^{2} / m_{0}^{2} \leq 10
\end{gathered}
$$

The values of $h_{e}^{G U T}, h_{\mu}^{G U T}, h_{\tau}^{G U T}$ are defined in such a way that we get the charged lepton masses correctly. As the charginos mix with the leptons, through a mass matrix given by

$\mathcal{M}_{C}=\left[\begin{array}{cc}M_{C} & A \\ B & M_{L}\end{array}\right]$

where $M_{C}$ is the usual MSSM chargino mass matrix,

$$
M_{C}=\left[\begin{array}{cc}
M & \frac{1}{\sqrt{2}} g v_{u} \\
\frac{1}{\sqrt{2}} g v_{d} & \mu
\end{array}\right]
$$

$M_{L}$ is the lepton mass matrix, that we consider diagonal,

$$
M_{L}=\left[\begin{array}{ccc}
\frac{1}{\sqrt{2}} h_{E_{11}} v_{d} & 0 & 0 \\
0 & \frac{1}{\sqrt{2}} h_{E_{22}} v_{d} & 0 \\
0 & 0 & \frac{1}{\sqrt{2}} h_{E_{33}} v_{d}
\end{array}\right]
$$

and $A$ and $B$ are matrices that are non zero due to the violation of $R_{P}$ and are given by

$$
A^{T}=\left[\begin{array}{cc}
-\frac{1}{2} h_{E_{11}} v_{1} & 0 \\
-\frac{1}{2} h_{E_{22}} v_{1} & 0 \\
-\frac{1}{2} h_{E_{33}} v_{3} & 0
\end{array}\right] B=\left[\begin{array}{cc}
\frac{1}{2} g v_{3} & -\epsilon_{1} \\
\frac{1}{2} g v_{3} & -\epsilon_{2} \\
\frac{1}{2} g v_{3} & -\epsilon_{3}
\end{array}\right]
$$

We used an iterative procedure to accomplish that the three lightest eigenvalues of $\mathcal{M}_{C}$ are in
Table 2

Counting of free parameters in MSSM

\begin{tabular}{ccc}
\hline Parameters & Conditions & Free Parameters \\
\hline$h_{t}, h_{b}, h_{\tau}$ & $m_{W}, m_{t}$ & $\tan \beta$ \\
$v_{d}, v_{u}, M_{1 / 2}$ & $m_{b}, m_{\tau}$ & 2 Extra \\
$m_{0}, A, \mu$ & $t_{i}=0, i=1,2$ & $\left(\right.$ e.g. $\left.m_{h}, m_{A}\right)$ \\
\hline Total $=9$ & Total $=6$ & Total $=3$ \\
\hline
\end{tabular}

Table 3

Counting of free parameters in our model

\begin{tabular}{ccc}
\hline Parameters & Conditions & Free Parameters \\
\hline$h_{t}, h_{b}, h_{\tau}$ & $m_{W}, m_{t}$ & $\tan \beta, \epsilon_{i}$ \\
$v_{d}, v_{u}, M_{1 / 2}$ & $m_{b}, m_{\tau}$ & \\
$m_{0}, A, \mu$ & $t_{i}=0$ & 2 Extra \\
$v_{i}, \epsilon_{i}$ & $(i=1, \ldots, 5)$ & $\left(\right.$ e.g. $\left.m_{h}, m_{A}\right)$ \\
\hline Total $=15$ & Total $=9$ & Total $=6$ \\
\hline
\end{tabular}

agreement with the experimental masses of the leptons. After running the RGE we have a complete set of parameters, Yukawa couplings and soft-breaking masses $m_{i}^{2}(R G E)$ to study the minimization. This is done by the following method: we solve the minimization equations for the soft masses squared. This is easy because those equations are linear on the soft masses squared. The values obtained in this way, that we call $m_{i}^{2}$ are not equal to the values $m_{i}^{2}(R G E)$ that we got via RGE. To achieve equality we define a function

$\eta=\max \left(\frac{m_{i}^{2}}{m_{i}^{2}(R G E)}, \frac{m_{i}^{2}(R G E)}{m_{i}^{2}}\right) \quad \forall i$

with the obvious property that

$\eta \geq 1$

Then we adjust the parameters to minimize $\eta$.

Before we end this section let us discuss the counting of free parameters in this model and in the minimal $\mathrm{N}=1$ supergravity unified version of the MSSM. In Table 2 we show this counting for the MSSM and in Table 3 for the $\epsilon$-model. Finally, we note that in either case, the sign of the mixing parameter $\mu$ is physical and has to be taken into account. 


\subsection{Gauge and Yukawa Unification in the $\epsilon$ model}

There is a strong motivation to consider GUT theories where both gauge and Yukawa unification can achieved. This is because besides achieving gauge coupling unification, GUT theories also reduce the number of free parameters in the Yukawa sector and this is normally a desirable feature. The situation with respect to the MSSM can be summarized as follows:

- In $S U(5)$ models, $h_{b}=h_{\tau}$ at $M_{G U T}$. The predicted ratio $m_{b} / m_{\tau}$ at $M_{W E A K}$ agrees with experiments.

- A relation between $m_{t o p}$ and $\tan \beta$ is predicted. Two solutions are possible: low and high $\tan \beta$.

- In $S O(10)$ and $E_{6}$ models $h_{t}=h_{b}=h_{\tau}$ at $M_{G U T}$. In this case, only the large $\tan \beta$ solution survives.

We have shown [19] that the $\epsilon$-model allows $b-\tau$ Yukawa unification for any value of $\tan \beta$ and satisfying perturbativity of the couplings. We also find the $t-b-\tau$ Yukawa unification easier to achieve than in the MSSM, occurring in a wider high $\tan \beta$ region.

\section{Tree Level Neutrino Masses and Mix- ings}

\subsection{Neutral fermion mass matrix}

In the basis

$\psi^{0 T}=\left(-i \lambda^{\prime},-i \lambda^{3}, \widetilde{H}_{d}^{1}, \widetilde{H}_{u}^{2}, \nu_{e}, \nu_{\mu}, \nu_{\tau}\right)$

the neutral fermions mass terms in the Lagrangian are given by

$\mathcal{L}_{m}=-\frac{1}{2}\left(\psi^{0}\right)^{T} \boldsymbol{M}_{N} \psi^{0}+$ h.c.

where the neutralino/neutrino mass matrix is

$\boldsymbol{M}_{N}=\left[\begin{array}{cc}\mathcal{M}_{\chi^{0}} & m^{T} \\ m & 0\end{array}\right]$

with

$$
\mathcal{M}_{\chi^{0}}=\left[\begin{array}{cccc}
M_{1} & 0 & -\frac{1}{2} g^{\prime} v_{d} & \frac{1}{2} g^{\prime} v_{u} \\
0 & M_{2} & \frac{1}{2} g v_{d} & -\frac{1}{2} g v_{u} \\
-\frac{1}{2} g^{\prime} v_{d} & \frac{1}{2} g v_{d} & 0 & -\mu \\
\frac{1}{2} g^{\prime} v_{u} & -\frac{1}{2} g v_{u} & -\mu & 0
\end{array}\right]
$$

$$
m=\left[\begin{array}{cccc}
-\frac{1}{2} g^{\prime} v_{1} & \frac{1}{2} g v_{1} & 0 & \epsilon_{1} \\
-\frac{1}{2} g^{\prime} v_{2} & \frac{1}{2} g v_{2} & 0 & \epsilon_{2} \\
-\frac{1}{2} g^{\prime} v_{3} & \frac{1}{2} g v_{3} & 0 & \epsilon_{3}
\end{array}\right]
$$

The mass matrix $\boldsymbol{M}_{N}$ is diagonalized by

$\mathcal{N}^{*} \boldsymbol{M}_{N} \mathcal{N}^{-1}=\operatorname{diag}\left(m_{\chi_{i}^{0}}, m_{\nu_{j}}\right)$

where $(i=1, \cdots, 4)$ for the neutralinos, and $(j=$ $1, \cdots, 3)$ for the neutrinos.

\subsection{Approximate diagonalization of mass matrices}

If the $R_{p}$ parameters are small it is convenient to define [20] the matrix

$\xi=m \cdot \mathcal{M}_{\chi^{0}}^{-1}$

If the elements of this matrix satisfy

$\forall \xi_{i j} \ll 1$

then one can find an approximate solution for mixing matrix $\mathcal{N}$. Explicitly we have

$$
\begin{aligned}
\xi_{i 1} & =\frac{g^{\prime} M_{2} \mu}{2 \operatorname{det}\left(\mathcal{M}_{\chi^{0}}\right)} \Lambda_{i} \\
\xi_{i 2} & =-\frac{g M_{1} \mu}{2 \operatorname{det}\left(\mathcal{M}_{\chi^{0}}\right)} \Lambda_{i} \\
\xi_{i 3} & =-\frac{\epsilon_{i}}{\mu}+\frac{\left(g^{2} M_{1}+g^{\prime 2} M_{2}\right) v_{2}}{4 \operatorname{det}\left(\mathcal{M}_{\chi^{0}}\right)} \Lambda_{i} \\
\xi_{i 4} & =-\frac{\left(g^{2} M_{1}+g^{\prime 2} M_{2}\right) v_{1}}{4 \operatorname{det}\left(\mathcal{M}_{\chi^{0}}\right)} \Lambda_{i}
\end{aligned}
$$

where

$\Lambda_{i}=\mu v_{i}+v_{d} \epsilon_{i}$

From Eq. (51) and Eq. (52) one can see that $\xi=0$ in the MSSM limit where $\epsilon_{i}=0, v_{i}=0$. In leading order in $\xi$ the mixing matrix $\mathcal{N}$ is given by,

$\mathcal{N}^{*}=\left(\begin{array}{cc}N^{*} & 0 \\ 0 & V_{\nu}^{T}\end{array}\right)\left(\begin{array}{cc}1-\frac{1}{2} \xi^{\dagger} \xi & \xi^{\dagger} \\ -\xi & 1-\frac{1}{2} \xi \xi^{\dagger}\end{array}\right)$

The second matrix above block-diagonalizes $\boldsymbol{M}_{N}$ approximately to the form $\operatorname{diag}\left(m_{e f f}, \mathcal{M}_{\chi^{0}}\right)$

$m_{e f f}=-m \cdot \mathcal{M}_{\chi^{0}}^{-1} m^{T}$ 


$$
=\frac{M_{1} g^{2}+M_{2} g^{\prime 2}}{4 \operatorname{det}\left(\mathcal{M}_{\chi^{0}}\right)}\left(\begin{array}{ccc}
\Lambda_{e}^{2} & \Lambda_{e} \Lambda_{\mu} & \Lambda_{e} \Lambda_{\tau} \\
\Lambda_{e} \Lambda_{\mu} & \Lambda_{\mu}^{2} & \Lambda_{\mu} \Lambda_{\tau} \\
\Lambda_{e} \Lambda_{\tau} & \Lambda_{\mu} \Lambda_{\tau} & \Lambda_{\tau}^{2}
\end{array}\right)
$$

The sub-matrices $N$ and $V_{\nu}$ in eq. diagonalize $\mathcal{M}_{\chi^{0}}$ and $m_{\text {eff }}$

$N^{*} \mathcal{M}_{\chi^{0}} N^{\dagger}=\operatorname{diag}\left(m_{\chi_{i}^{0}}\right)$,

$V_{\nu}^{T} m_{e f f} V_{\nu}=\operatorname{diag}\left(0,0, m_{\nu}\right)$

where

$m_{\nu}=\operatorname{Tr}\left(m_{e f f}\right)=\frac{M_{1} g^{2}+M_{2}{g^{\prime}}^{2}}{4 \operatorname{det}\left(\mathcal{M}_{\chi^{0}}\right)}|\vec{\Lambda}|^{2}$.

For $V_{\nu}$ we have ( we can rotate away one angle)

$$
\begin{aligned}
V_{\nu}= & \left(\begin{array}{ccc}
1 & 0 & 0 \\
0 & \cos \theta_{23} & -\sin \theta_{23} \\
0 & \sin \theta_{23} & \cos \theta_{23}
\end{array}\right) \times \\
& \left(\begin{array}{ccc}
\cos \theta_{13} & 0 & -\sin \theta_{13} \\
0 & 1 & 0 \\
\sin \theta_{13} & 0 & \cos \theta_{13}
\end{array}\right),
\end{aligned}
$$

where the mixing angles can be expressed in terms of the alignment vector $\vec{\Lambda}$ as follows:

$$
\begin{aligned}
& \tan \theta_{13}=-\frac{\Lambda_{e}}{\left(\Lambda_{\mu}^{2}+\Lambda_{\tau}^{2}\right)^{\frac{1}{2}}}, \\
& \tan \theta_{23}=\frac{\Lambda_{\mu}}{\Lambda_{\tau}} .
\end{aligned}
$$

\section{One Loop Neutrino Masses and Mixings}

\subsection{Definition}

The Self-Energy for the neutralino/neutrino is

$$
\begin{aligned}
\vec{i} \bigcirc \rightarrow_{j} \equiv & i\left\{/ p\left[P_{L} \Sigma_{i j}^{L}+P_{R} \Sigma_{i j}^{R}\right]\right. \\
& \left.-\left[P_{L} \Pi_{i j}^{L}+P_{R} \Pi_{i j}^{R}\right]\right\}(61)
\end{aligned}
$$

Then

$M_{i j}^{\text {pole }}=M_{i j}^{\overline{\mathrm{DR}}}\left(\mu_{R}\right)+\Delta M_{i j}$

where

$$
\Delta M_{i j}=\left[\frac{1}{2}\left(\Pi_{i j}^{V}\left(m_{i}^{2}\right)+\Pi_{i j}^{V}\left(m_{j}^{2}\right)\right)\right.
$$
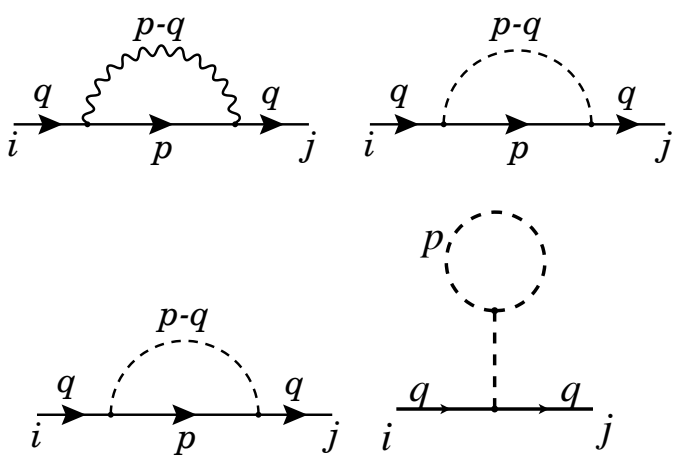

Figure 2. Diagrams contributing

$$
\left.-\frac{1}{2}\left(m_{\chi_{i}^{0}} \Sigma_{i j}^{V}\left(m_{i}^{2}\right)+m_{\chi_{j}^{0}} \Sigma_{i j}^{V}\left(m_{j}^{2}\right)\right)\right]_{\Delta=0}(63)
$$

where

$\Sigma^{V}=\frac{1}{2}\left(\Sigma^{L}+\Sigma^{R}\right) \quad \Pi^{V}=\frac{1}{2}\left(\Pi^{L}+\Pi^{R}\right)$

and

$\Delta=\frac{2}{4-d}-\gamma_{E}+\ln 4 \pi$

\subsection{Diagrams Contributing}

In Fig. (2) are shown the classes of diagrams contributing to the self-energy at one loop. These diagrams can be calculated in a straightforward way. For instance the $W$ diagram in the $\xi=1$ gauge gives

$$
\begin{gathered}
\Sigma_{i j}^{V}=-\frac{1}{16 \pi^{2}} \sum_{k=1}^{5} 2\left(O_{L j k}^{\mathrm{ncw}} O_{L k i}^{\mathrm{cnw}}+O_{R j k}^{\mathrm{ncw}} O_{R k i}^{\mathrm{cnw}}\right) \\
B_{1}\left(p^{2}, m_{k}^{2}, m_{W}^{2}\right) \\
\Pi_{i j}^{V}=-\frac{1}{16 \pi^{2}} \sum_{k=1}^{5}(-4)\left(O_{L j k}^{\mathrm{ncw}} O_{R k i}^{\mathrm{cnw}}+O_{R j k}^{\mathrm{ncw}} O_{L k i}^{\mathrm{cnw}}\right)
\end{gathered}
$$

$$
m_{k} B_{0}\left(p^{2}, m_{k}^{2}, m_{W}^{2}\right)
$$

where $B_{0}$ and $B_{1}$ are the Passarino-Veltman functions, and the coupling matrices appear in the vertices in the following way 


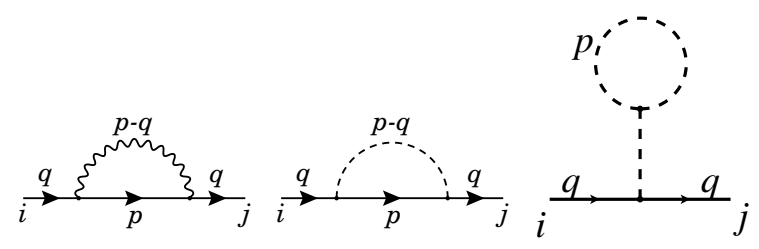

Figure 3. Set 1
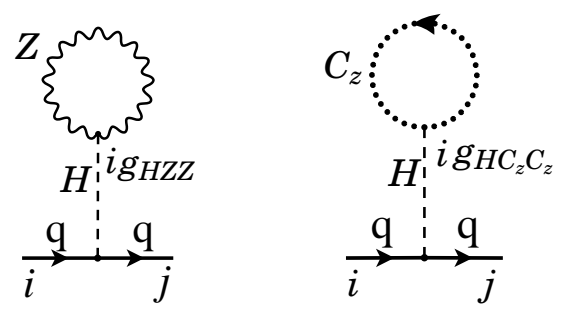

Figure 4. Set 2

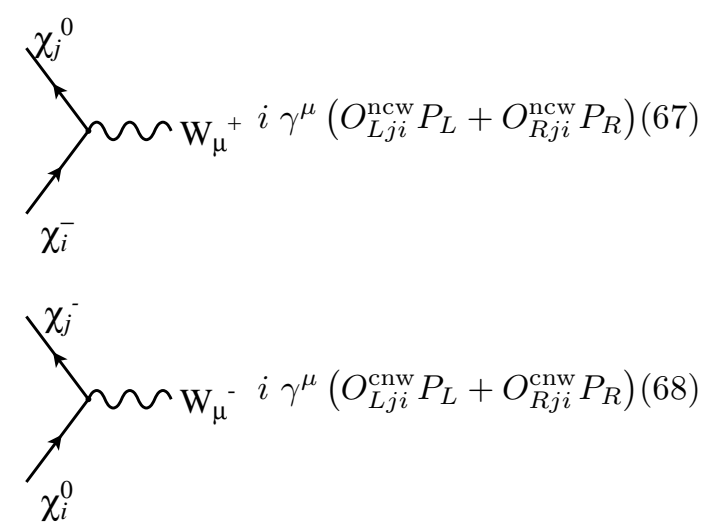

\subsection{Gauge Invariance}

When calculating the self-energies the question of gauge invariance arises. In the $R_{\xi}$ gauge the sets of diagrams of Figs. 35 depend on $\xi$. We have shown that the gauge dependence cancels among the diagrams in each set. So in the actual

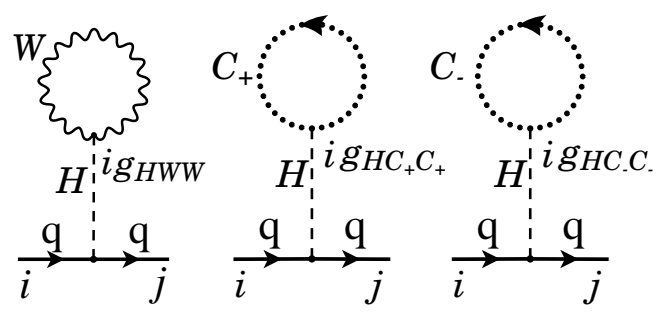

Figure 5. Set 3 calculations we considered the tadpoles needed in those sets to ensure gauge invariance. The other tadpoles were included in the minimization procedure of Eq. (34). This is a gauge invariant splitting.

\subsection{The One-Loop Mass Matrix}

The one-loop corrected mass matrix is given by

$M^{1 L}=M_{\text {diag }}^{0 L}+\Delta M^{1 L}$

where

$M_{\text {diag }}^{0 L}=\mathcal{N} M_{N} \mathcal{N}^{T}$

Now we diagonalize the 1-loop mass matrix

$M_{\text {diag }}^{1 L}=\mathcal{N}^{\prime} M^{1 L} \mathcal{N}^{\prime T}$

Then the mass eigenstates are related to the weak basis states by

$\chi_{0}^{\text {mass }}=\mathcal{N}_{i \alpha}^{1 L} \chi_{0}^{\text {weak }}$

with

$\mathcal{N}^{1 L}=\mathcal{N}^{\prime} \mathcal{N}$

The usual convention in neutrino physics

$\nu_{\alpha}=U_{\alpha k} \nu_{k}$

is recovered in our notation as

$U_{\alpha k}=\mathcal{N}_{4+k, 4+\alpha}^{1 L}$

\subsection{Solar and Atmospheric Neutrino Pa- rameters}

Assuming hierarchy in the masses $m_{\nu_{2}}$ and $m_{\nu_{3}}$ the survival probabilities for the solar and atmospheric neutrinos are

$P_{e}=1-4 U_{e 1}^{2} U_{e 2}^{2} \sin ^{2}\left(\frac{\Delta m_{21}^{2} t}{4 E}\right)-2 U_{e 3}^{2}\left(1-U_{e 3}^{2}\right)$

$P_{\mu}=1-4 U_{\mu 3}^{2}\left(1-U_{\mu 3}^{2}\right) \sin ^{2}\left(\frac{\Delta m_{21}^{2} t}{4 E}\right)$

As $U_{e 3}$ has to be small we neglect it and write the usual two neutrino mixing angle as

$\sin ^{2}\left(2 \theta_{12}\right)=4 U_{e 1}^{2} U_{e 2}^{2}$

and

$\sin ^{2}\left(2 \theta_{13}\right)=4 U_{\mu 3}^{2}\left(1-U_{\mu 3}^{2}\right)$ 


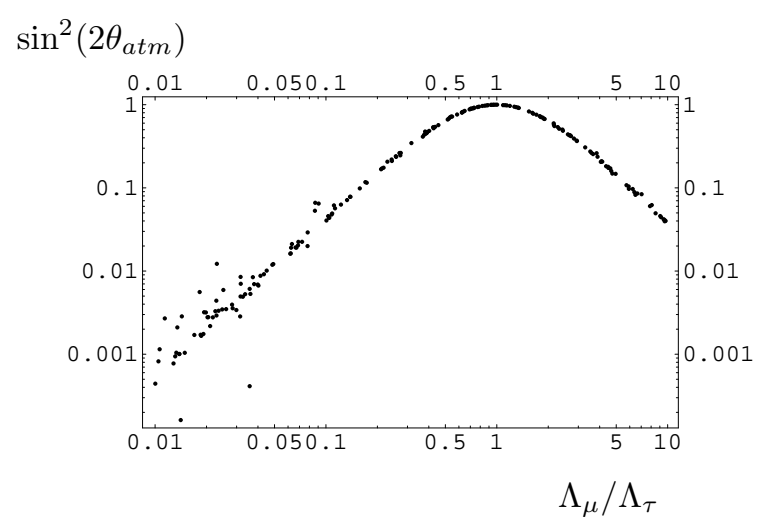

Figure 6. The atmospheric angle as function of $\Lambda_{\mu} / \Lambda_{\tau}$ for $\left|\epsilon_{i}\right|=\epsilon$ and $\Lambda_{e}=0.1 \Lambda_{\tau} . \epsilon^{2} / \Lambda$ has an upper cut of $\epsilon^{2} / \Lambda \leq 0.1$ in this plot, since larger values lead to larger scatter for very small $\Lambda_{\mu} / \Lambda_{\tau}$

\subsection{Our Preliminary Results}

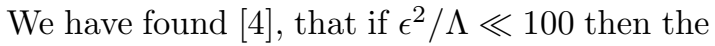
approximate formulas hold

$$
\begin{gathered}
U_{e 3} \approx \sin \left(\tan ^{-1}\left(\Lambda_{1} / \sqrt{\Lambda_{2}^{2}+\Lambda_{3}^{2}}\right)\right) \\
U_{\mu 3} \approx \sin \left(\tan ^{-1}\left(\Lambda_{2} / \sqrt{\Lambda_{1}^{2}+\Lambda_{3}^{2}}\right)\right) \\
U_{\tau 3} \approx \sin \left(\tan ^{-1}\left(\Lambda_{3} / \sqrt{\Lambda_{1}^{2}+\Lambda_{2}^{2}}\right)\right)
\end{gathered}
$$

Then if we take

$\Lambda_{1} \ll \Lambda_{2} \simeq \Lambda_{3}$

we immediately get maximal mixing for the atmospheric neutrinos. This is shown in Figure 6 where we see that maximality of the mixing is only possible for $\Lambda_{\mu}=\Lambda_{\tau}$.

To get bimaximality we have to fix the solar angle. We have discovered that if $\epsilon_{e} \simeq \epsilon_{\mu} \simeq \epsilon_{\tau}$ and $\Lambda_{e} \ll \Lambda_{\mu} \simeq \Lambda_{\tau}$ we get bimaximality if the following sign condition applies

$\left(\epsilon_{\mu} / \epsilon_{\tau}\right) \times\left(\Lambda_{\mu} / \Lambda_{\tau}\right) \leq 0$

This is illustrated in Figure 7. In practice we do not need perfect maximality. We took

$$
4 / 5 \leq\left|\Lambda_{\mu} / \Lambda_{\tau}\right| \leq 5 / 4 \quad \text { and } \quad\left|\Lambda_{e} / \Lambda_{\mu}\right| \ll 1
$$

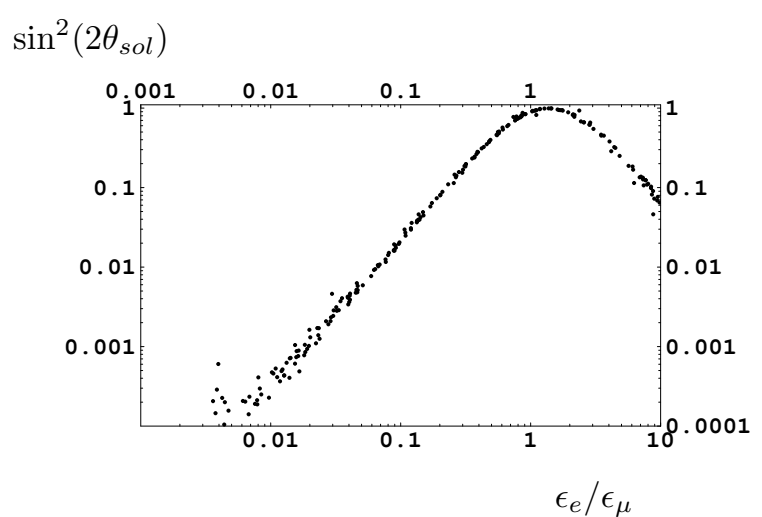

Figure 7. The solar angle as function of $\epsilon_{e} / \epsilon_{\mu}$ for $\epsilon_{\mu}=\epsilon_{\tau}$ and $\Lambda_{\mu}=\Lambda_{\tau}$ applying the sign condition.

to fix the atmospheric angle and

$\left(\epsilon_{\mu} / \epsilon_{\tau}\right) \times\left(\Lambda_{\mu} / \Lambda_{\tau}\right) \leq 0$ and $0.6 \leq \epsilon_{e} / \epsilon_{\mu} \leq 1.2(83)$

to fix the solar angle. Next we have to fix the masses to solve the atmospheric and solar neutrino problems. We found [4 20] that the range

$0.03 \mathrm{GeV}^{2} \leq|\Lambda| \leq 0.25 \mathrm{GeV}^{2}$

fixes the tree level mass to reproduce the atmospheric neutrino problem. This is illustrated in Figure 8 where, besides the conditions in Eqs. 82) and (83) to fix the angles and condition Eq. (84), all the other parameters were chosen randomly. Consistency of the parameters was required in the sense that minimization the scalar potential including the tadpoles was performed as well as the matching with the RGE solutions with universality at GUT scale.

Finally we have to check if it is possible to have masses in the range to solve the solar neutrino problem. We found [4] that the relevant parameter for this purpose is

$\left(\epsilon_{1}^{2}+\epsilon_{1}^{2}+\epsilon_{1}^{2}\right) /|\Lambda| \equiv \epsilon^{2} /|\Lambda|$

Depending on this quantity in the range $0.01 \leq$ $\epsilon^{2} /|\Lambda| \leq 1$ the solar neutrino problem can be solved: Low values give just-so solutions, high values tend to give large angle MSW (LA-MSW). This is illustrated in Figure 9 and in Figure 10. Another question of relevance that we addressed was the study of the decay length of the lightest 


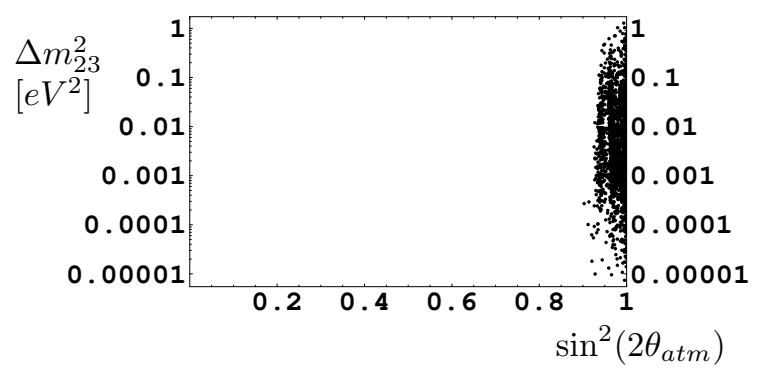

Figure 8. $\Delta m_{23}^{2}$ versus $\sin ^{2}\left(2 \theta_{a t m}\right)$. All points obey Eqs. (82), 83 and (84) no further cut applied except that $0.3 \leq \epsilon^{2} /|\Lambda| \leq 1$.

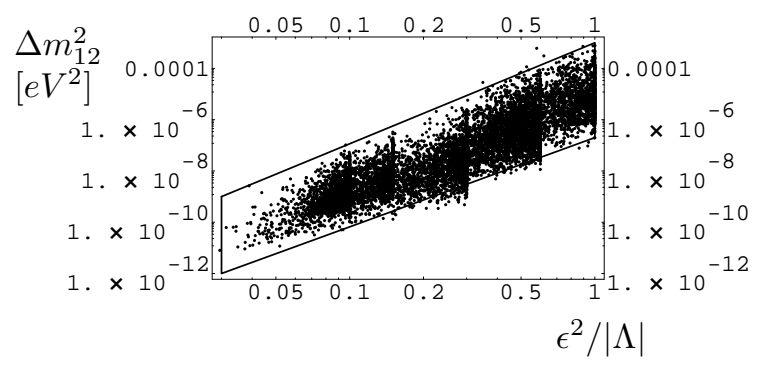

Figure 9. $\Delta m_{12}^{2}$ as a function of $\epsilon^{2} /|\Lambda|$. A box is drawn to guide the eye.

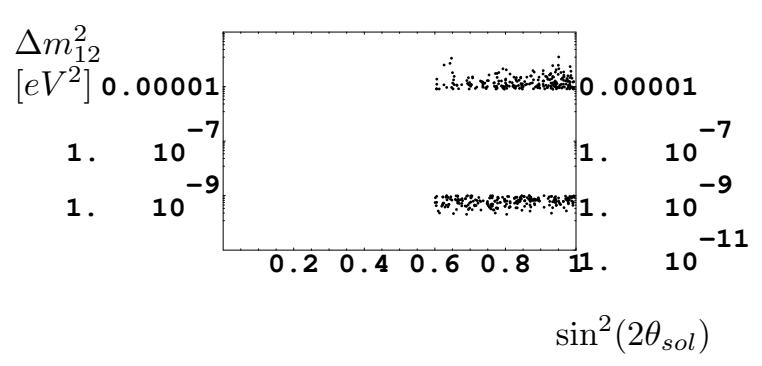

Figure 10. $\Delta m_{12}^{2}$ versus $\sin ^{2}\left(2 \theta_{\text {sol }}\right)$ for those points which have $\Delta m_{23}^{2}$ and $\sin ^{2}\left(2 \theta_{\text {atm }}\right)$ correct and at the same time fit either the vacuum or LA-MSW solutions.

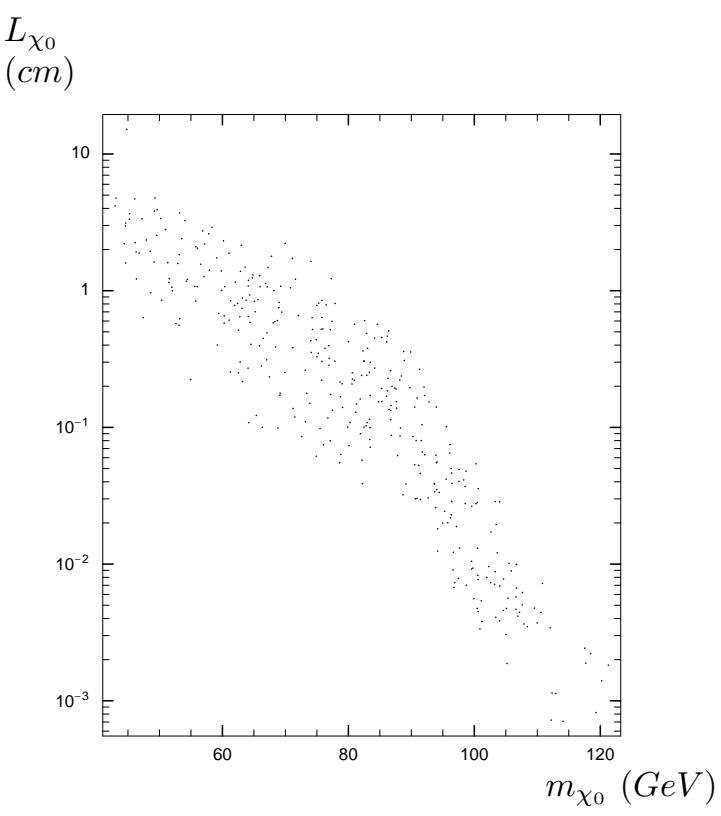

Figure 11. Neutralino decay length in $\mathrm{cm}$

neutralino. This is important, because if the decay length is greater than the detector, then in practice it will be invisible like in the MSSM. As we can see in Figure 11, that is not the case, the neutralino decays well inside the detector leading to novel signatures.

\section{Conclusions}

There is a viable model for SBRP that leads to a very rich phenomenology, both at laboratory experiments, and at present (LEP) and future (LHC, LNC) accelerators. We have shown that the radiative breaking of both the Gauge Symmetry and $R_{P}$ can be achieved. In these type of models neutrinos have mass and can decay thus avoiding the critical density argument. They also can evade the $\mathrm{BBN}$ limits on a $\nu_{\tau}$ on the $\mathrm{MeV}$ scale. Most of these phenomenology can be described by an effective model with bilinear explicit $R_{P}$ violation. We have calculated the oneloop corrected masses and mixings for the neutrinos in a completely consistent way, including the RG equations and correctly minimizing the potential. We have shown that it is possible to 
get bimaximal solutions for both the atmospheric and solar neutrino problems. We emphasize that the lightest neutralino decays inside the detectors, thus leading to a very different phenomenology than the MSSM.

\section{REFERENCES}

1. Yu. A. Gol'fand and E. P. Likhtman, Sov. Phys. JETP Lett. 13 (1971) 323; D.V. Volkov and V.P. Akulov, Sov. Phys. JETP Lett. 16 (1972) 438; J. Wess and B. Zumino, Nucl. Phys. B 70 (1974) 39.

2. H.P. Nilles, Phys. Rep. 110 (1984) 1; H.E. Haber and G.L. Kane, Phys. Rep. 117 (1985) 75; R. Barbieri, Riv. Nuovo Cimento 11 (1988) 1.

3. For a review see e.g J. C. Romão, Lectures given at the 5th Gleb Wataghin School on High-Energy Phenomenology, Campinas, Brazil, 13-17 Jul 1998. e-Print Archive: hep$\mathrm{ph} / 9811454$

4. M.A. Díaz, M. Hirsch, W. Porod, J.C. Romão and J.W.F. Valle in preparation.

5. M.A. Díaz, J.C. Romão, and J.W.F. Valle, Nucl. Phys. B 524 (1998) 23; M.A. Díaz, talk given at International Europhysics Conference on High Energy Physics, Jerusalem, Israel, 19-26 Aug 1997, hep-ph/9712213; J.C. Romão, talk given at International Workshop on Physics Beyond the Standard Model: From Theory to Experiment (Valencia 97), Valencia, Spain, 13-17 Oct 1997, hep-ph/9712362; J.W.F. Valle, review talk given at the Workshop on Physics Beyond the Standard Model: Beyond the Desert: Accelerator and Nonaccelerator Approaches, Tegernsee, Germany, 814 Jun 1997, hep-ph/9712277.

6. F. de Campos, M.A. García-Jareño, A.S. Joshipura, J. Rosiek, and J. W. F. Valle, Nucl. Phys. B 451 (1995) 3;T. Banks, Y. Grossman, E. Nardi, and Y. Nir, Phys. Rev. D 52 (1995) 5319; A. S. Joshipura and M.Nowakowski, Phys. Rev. D 51 (1995) 2421; R. Hempfling, Nucl. Phys. B 478 (1996) 3; F. Vissani and A.Yu. Smirnov, Nucl. Phys. B 460 (1996) 37; H. P. Nilles and N. Polonsky, Nucl. Phys. B 484 (1997) 33; B. de Carlos,
P. L. White, Phys. Rev. D 55 (1997) 4222; S. Roy and B. Mukhopadhyaya, Phys. Rev. D 55 (1997) 7020.

7. A. Akeroyd, M.A. Díaz, J. Ferrandis, M.A. Garcia-Jareño, and Jose W.F. Valle, Nucl. Phys. B 529 (1998) 3.

8. C Aulakh, R Mohapatra, Phys. Lett. B 119 (1983) 136; A Santamaria, J W F Valle, Phys. Lett. B 195 (1987) 423; Phys. Rev. Lett. 60 (1988) 397; Phys. Rev. D 39 (1989) 1780.

9. A Masiero, J W F Valle, Phys. Lett. B 251 (1990) 273.

10. A. Masiero and J.W.F. Valle, Phys. Lett. B 251 (1990) 273; J.C. Romão, C.A. Santos, J.W.F. Valle, Phys. Lett. B 288 (1992) 311;

11. A Lopez-Fernandez, J. Romão, F. de Campos and J. W. F. Valle, Phys. Lett. B 312 (1993) 240; ibidem, Proceedings of Moriond '94. pag.81-86, edited by J. Tran Thanh Van, Éditions Frontiéres, 1994. J. C. Romão, F. de Campos, M. A. Garcia-Jareno, M. B. Magro and J. W. F. Valle, Nucl. Phys. B 482 (1996) 3.

12. J C Romão, N Rius and J W F Valle, Nucl. Phys. B 363 (1991) 369.

13. P Nogueira, J C Romão, J W F Valle, Phys. Lett. B 251 (1990) 142.

14. J. C. Romão, J. W. F. Valle, Nucl. Phys. B 381 (1992) 87.

15. S. Bertolini and G. Steigman, Nucl. Phys. B 387 (1990) 193; M. Kawasaki et al, Nucl. Phys. B 402 (1993) 323; Nucl. Phys. B 419 (1994) 105; S. Dodelson, G. Gyuk and M.S. Turner, Phys. Rev. D 49 (1994) 5068.

16. A. D. Dolgov, S. Pastor, J.C. Romão and J. W. F. Valle, Nucl. Phys. B 496 (1997) 24.

17. M.A. Díaz, talk given at the International Workshop on Physics Beyond the Standard Model: From Theory to Experiment (Valencia 97), Valencia, Spain, 13-17 Oct 1997, hep-ph/9802407.

18. R. Hempfling, Nucl. Phys. B 478 (1996) 3.

19. M.A. Díaz, J. Ferrandis, J.C. Romão, and J.W.F. Valle, Phys. Lett. B 453 (1999) 263.

20. M. Hirsch, J.W.F. Valle, e-Print Archive: hep-ph/9812463. 